\title{
生物活性小分子与靶点相互作用研究中的 新方法和新技术
}

\section{唐功利 麻锦彪 吴厚铭“ 陈海宝"}

(中国科学院上海有机化学研究所生命有机化学国家重点实验室, 上海 200032.* 联系人, Email: hmwu@ pub.sioc.ac.cn; hbchen@pub.sioc.ac.cn)

摘要 活性小分子与靶蛋白的相互作用是生命中基本的相互作用之一, 因而靶蛋白和活性小分子 的篮选是生命有机化学及药物化学研究的重要内容. 在活性小分子篮选方面, 细胞印迹 (cytoblot)、以核磁共振为基础的结构活性关系研究(SAR-by-NMR)及反双杂交(reverse two-hybrid) 系统分别是以细胞、靶蛋白及蛋白间的相互作用为靶点的高通量、快速的篮选方法. 另外, 在由 活性小分子鉴定靶蛋白的研究中, 三杂交(three-hybrid)系统、功能表达克隆(functional expressioncloning) 及药物印迹(drug-western)等方法大大加速了靶蛋白的鉴定进程; 而展示克隆 (display cloning)方法可以直接完成由活性小分子到靶蛋白基因的克隆. 结合 DNA 芯片技术, 以基因组为 基础的篮选方法同时还可以明确小分子参与的一系列生物大分子调控. 而小分子印迹 (small molecular printing)是从组合化学出发适应人类基因组要求的篎选靶蛋白及活性小分子、研究小分 子参与调控的方法. 概要介绍这几种最新篮选方法, 并对中草药现代化进行了初步探讨.

关键词 靶蛋白 活性小分子 篮选 细胞印迹 SAR-by-NMR 反双杂交 三杂交 功能表达克隆 药物印迹 展示克隆 小分子印迹 化学基因学 中草药现代化

20 世纪 90 年代, 随着分子生物学和有机化学的飞速发展, 人类基因组计划的全面展开, 越来越多的有机化学家尝试通过活性有机小分子来探索生物大分子的功能及调控, 从而从分 子及化学键的水平揭示生命活动的本质. 于是一门融合了化学及生物学的交叉学科一一化学 生物学应运而生. 与此同时, 随着人类基因组学及蛋白组学的发展, 以活性有机小分子为探针 来研究基因表达及细胞周期调控的化学基因学(chemical genetics $)^{[1 \sim 4]}$ 正逐步形成. 在这些研究 中, 从生物活性小分子出发寻找它们的生物靶分子, 或从生物靶蛋白出发寻找高亲和性配体 分子, 是当前研究活性小分子与生物靶分子相互作用、分子识别、信息传递、生命过程的小 分子调控机制及发现新颖药物的关键环节. 最近这一领域出现了一系列新概念、新方法和新 技术, 如细胞印迹, SAR-by-NMR, 反双杂交技术, 三杂交系统, 功能表达克隆, 药物印迹, 展 示克隆, DNA 芯片技术及小分子印迹等. 本文结合化学基因学, 主要介绍这几种最新篮选方法 的原理、特点及在发现活性小分子和靶蛋白方面的应用.

\section{1 活性小分子的篮选}

有机化学, 特别是组合化学可以方便地创造数量极其庞大的小分子化合物库, 但是以往 如何从这庞大的化合物库中篮选到期望的活性分子却面临很大的困难, 缺少灵敏、有效且可 快速篮选的方法及手段是当时的主要障碍. 下面介绍的最近发展的几种高通量、快速而灵敏 的篮选活性小分子的方法也许是对克服上述困难的有益的探索.

\section{1 高通量活性小分子的篮选 一细胞印迹}

细胞印迹(cytoblot) $)^{[5]}$, 即高通量整细胞免疫检测(high-throughput whole-cell immunodetec- 
tion assay), 是在酶偶联免疫吸附测定(ELISA)和免疫印迹 (western blotting)的基础上发展起 来的. 该方法用于快速检测小分子对 DNA 合成、蛋白翻译后加工(乙酰化、磷酸化等)及细胞 周期等的影响. 其基本原理与免疫印迹十分类似(图 1), 但是检测某一类细胞中的分子是在多 孔培养板上的整细胞水平进行的. 首先以待检测的分子作为抗原制备抗体, 即一抗; 然后选 择合适的二抗进行检测, 如采用联有辣根过氧化酶的二抗与一抗偶联, 形成的复合物通过加 入氨基苯二酰一肼(luminol)、过氧化氢和对碘苯酚进行检测. 若细胞中有待检测的分子(即抗 原)存在, 则引发的化学发光反应使底片感光; 若不存在, 则无发光反应. 5-溴尿嘧啶是一种化 学诱变剂, 在 DNA 复制过程中, 它的渗入可使原来的 A-T 配对转变为 G-C 配对, 从而导致 癌变. 凡是可以阻止 5-溴尿嘧啶渗入的化合物在一定程度上具有抗癌作用. Stockwell 等人 ${ }^{[5]}$ 采用细胞印迹法在 6144 孔细胞培养板上验证了已知的抗癌药如转化生长因子- $\beta$ (transforming growth factor $\beta$ )、噻氨酯哒唑(nocodazole)等阻止 5-溴尿嘧啶渗入 DNA 合成的情况, 以此建 立了细胞印迹篮选活性小分子的方法. 其最大的优点是能够进行活性小分子的高通量快速篮 选, 可以采用组织特异的细胞在纳升到毫升的规模培养. 但该方法一般适合于初篎, 要进一步 明确所得小分子的活性还需结合其他篮选方法.
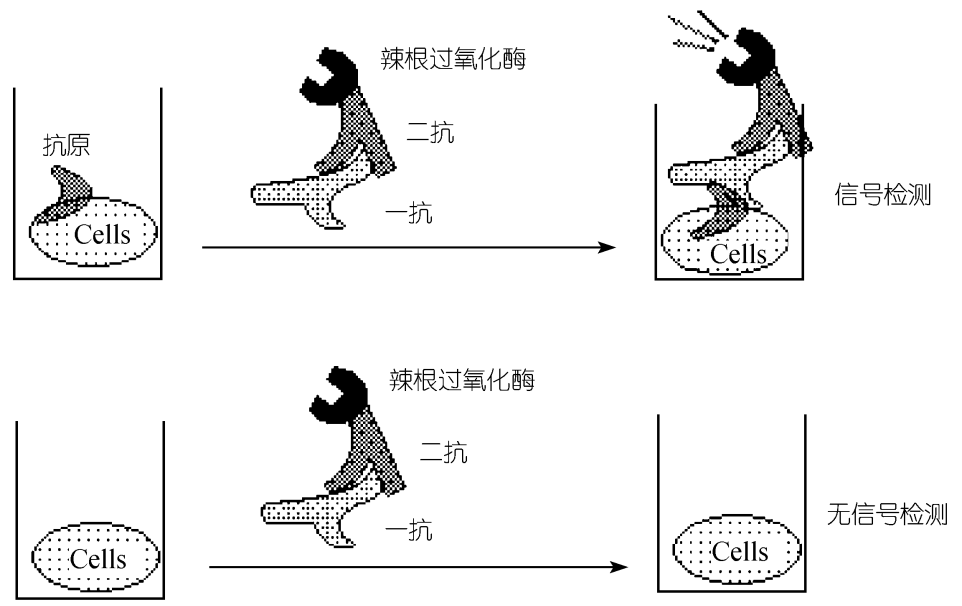

图 1 细胞印迹原理示意图

核仁素(nucleolin)是细胞核仁中的一种蛋白，当细胞进入有丝分裂时核仁素被特异地磷酸 化, 而可以抑制有丝分裂的小分子化合物均可以增加磷酸化核仁素的含量. 最近为了寻找不 与微管蛋白相互作用的有丝分裂抑制剂, Mayer 和 Haggarty 等人崖 ${ }^{[6]}$ 以磷酸化核仁素为抗原制 备一抗, 采用细胞印迹法从合成的 16320 种化合物中篮选到 139 种化合物可以阻止细胞分裂 (图 2). 然后, 采用体外微管蛋白的聚合检测, 排除其中 53 个与微管蛋白相互作用的分子得到 了 86 个化合物. 进一步采用体内染色体和微管染色检测等方法篮选到一种通过阻止纺锤体形 成而抑制细胞有丝分裂的活性化合物 Monastrol (图 2), 在该化合物的作用下正常纺锤体被星 状单极化. 结合他们及其他实验室工作的积累, 进一步推断该化合物很可能至少作用于有丝 分裂驱动蛋白 (mitotic kinesin)Eg5. 该活性分子作用机制完全不同于目前广泛研究的紫杉醇等 作用于微管蛋白而抑制有丝分裂的抗癌药, 其靶蛋白的确定为进一步合成、篮选 Monastrol 
的高活性类似物提供了篮选模型, 因此完全有可能发展成一类像紫杉醇一样有效但不具有神 经毒性、细胞毒性等副作用的新型抗癌药 ${ }^{[8]}$. 同时, 从该化合物的篎选到作用机理的阐明及靶蛋 白的鉴定首次提供了一种完全在实验室中从有机化学和生物学的角度开发新药的新思路 ${ }^{[9,10]}$.

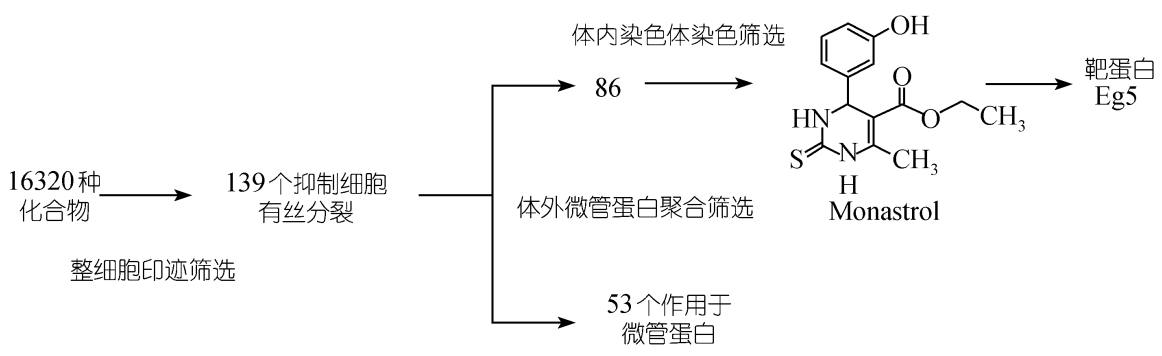

图 2 多步篮选获得新活性化合物 Monastrol

\section{2 以靶蛋白为模型篮选小分子配体—SAR-by-NMR}

在明确与疾病相关的靶蛋白的基础上, 人们利用现代生物工程结合 NMR 技术, 发展了一 种新的具有一定导向性的快速发现生物大分子高亲和性配体的方法—-SAR-by-NMR ${ }^{\mathrm{TM}}[11,12]$ (structure-activity relationship by nuclear magnetic resonance). 该方法结合了合理性设计中的配 体设计和优化方法和非合理性设计 (如组合化学) 的合理因素, 大大地提高了先导化合物发现 的速度和有效性. 其基本过程包括 5 个步骤(图 3) ${ }^{[12]}$ ：(1) 采用基因工程方法制备 ${ }^{15} \mathrm{~N}$ 标记的 靶蛋白, 通过二维 NMR 技术从某一小分子化合物库中篮选出和靶蛋白结合的第 1 个先导小 分子. (2) 对该分子的类似物进行篮选, 通过结构与活性的关系(SAR)对其进行优化. (3) 采用 与第 1 步相同的方法篮选出与前一结合亚位点相邻的亚位点结合的第 2 个先导分子. (4) 对第 2 个先导分子的类似物进行篮选, 得到第 2 个优化的先导分子. 在选定两个先导分子片段之后, 用多维 NMR ${ }^{[13}$, 14]等技术测定蛋白质和两个配体的复合物的完整三维空间结构, 确定两个配 体在靶蛋白上确切的结合位置及其空间取向. (5) 基于上述三维结构设计恰当的连接桥将两个 先导分子连接起来, 使得到的分子和靶蛋白结合时保持各自独立时的结合位置及其空间取向,

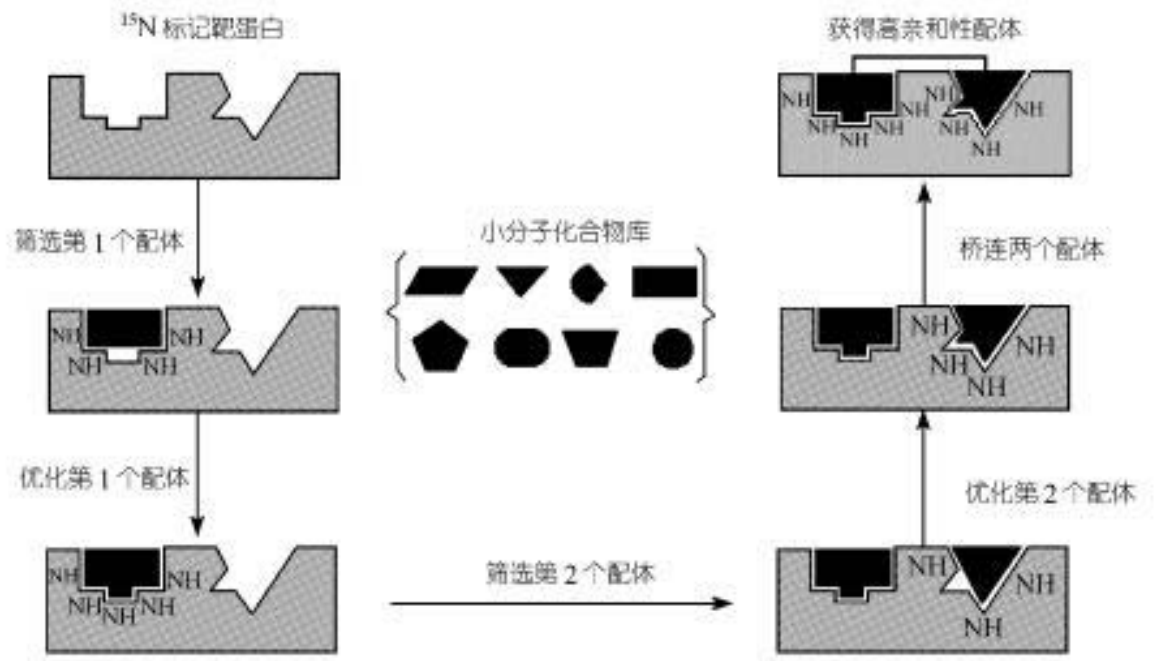

图 3 SAR-by-NMR 原理示意图 
最终篮选得到一个高亲和性的配体. 由于采用 NMR 技术可以综合多种药物设计的优势, 能 够在短时间内得到先导化合物, 而且在溶液中检测的是小分子与靶蛋白相互作用的真实情况, 因而大大加快了药物发现的速度. 到目前为止采用该方法已成功地发现了 FK506 结合蛋白的 高亲和性配体 ${ }^{[12]}$ 、溶基质素的非肽抑制剂 ${ }^{[15]}$ 、人乳头状病毒(papilloma virus)E2 蛋白的抑制 剂 ${ }^{[16]} \mathrm{SH}$ 结构域的高亲和性配体 ${ }^{[17]}$ 及 rRNA 甲基转移酶 ErmAM 的抑制剂 ${ }^{[18]}$ 等. 最近的研究表 明, 采用低温核磁共振探测技术 ${ }^{[19]}$, SAR-by-NMR 可用于活性小分子的快速、高通量篮选.

\section{3 利用靶蛋白-蛋白相互作用簃选活性小分子一一双杂交系统}

蛋白-蛋白的相互作用是生命活动中广泛而重要的相互作用, 与许多疾病相关, 因此以蛋 白-蛋白的相互作用为豨体篮选活性小分子是药物篮选的另一重要途径 ${ }^{[3,20]}$. 酵母双杂交技术 是研究蛋白与蛋白 (或多肽)间相互作用的常用技术 ${ }^{[21 ~ 23]}$, 而最近由此发展的反双杂交系统 (reverse two-hybrid system) 就是利用蛋白-蛋白的相互作用为靶体在细胞水平上来篮选活性小 分子的高通量簃选方法. 酵母双杂交技术的基本原理是融合于 DNA 结合域(DB)的蛋白 X 与 融合于转录活化域( $\mathrm{AD}$ )的蛋白 $\mathrm{Y}$ 的相互作用导致 $\mathrm{BD}$ 与 $\mathrm{AD}$ 空间上的接近, 从而激活报告基 因的转录及表达. 反双杂交基本原理与酵母双杂交技术相似(图 4), 只是报告基因所导致的细 胞生长表型正好相反, 即只有在 $\mathrm{X}$ 与 $\mathrm{Y}$ 不发生相互作用的情况下报告基因不被启动, 细胞才 能生长. 当采用与疾病有关的一对可发生相互作用的蛋白作为 $\mathrm{X}$ 与 $\mathrm{Y}$, 采用反双杂交系统来 篮选活性小分子时, 若小分子有活性, 抑制了 $\mathrm{X}$ 与 $\mathrm{Y}$ 的相互作用, 报告基因不被转录, 细胞 正常生长; 若待篮选小分子无活性, 不影响 $\mathrm{X}$ 与 $\mathrm{Y}$ 的相互作用, 这时启动报告基因的转录, 产 生的蛋白分解培养基中的某一特定化合物产生细胞毒性(如 $U R A 3^{[24]}$ 分解 5-氟乳清酸, 5-fluoroorotic acid), 酵母细胞就不能生长. 将转移生长因子- $\beta$ - I 受体作为反双杂交系统中的 $X$ 融合到 DB 上, FK506 结合蛋白 12(FKBP12)作为 $\mathrm{Y}$ 融合到 AD 上 ${ }^{[25]}$, 采用该系统可以检测到 FK506 的活 性 ${ }^{[3,25,26]}$. 最近, Young 等 ${ }^{[27]}$ 采用 $\mathrm{N}$ 型钲离子通道亚基间相互作用建立的反双杂交系统对 156000 个合成的化合物库进行了系统的篮选, 获得了一个较好的抑制 $\mathrm{N}$ 型钙离子通道的活性小分子, 它有可能发展成一类新型的、有潜在治疗意义的钙离子通道拮抗剂.

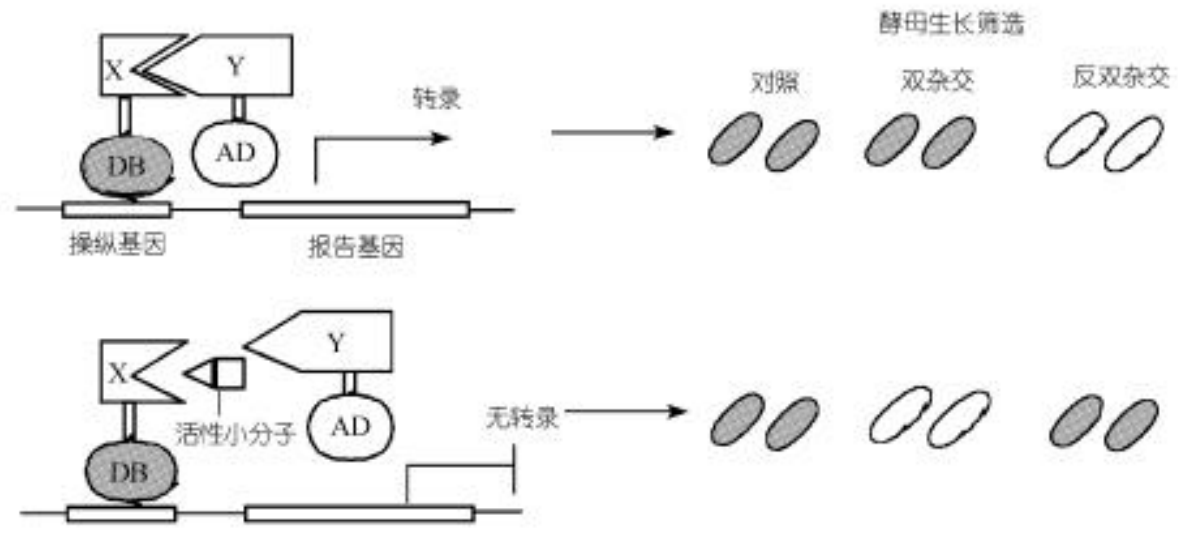

图 4 反双杂交系统篮选活性小分子原理示意图

图中右侧酵母生长情况的阴影斑代表酵母细胞正常生长, 空白斑代表酵母细胞没有生长或非正常生长. DB 代表 DNA 结合蛋白; AD 代表活化蛋白结构域 
由于该方法在细胞水平篮选, 因此小分子的通透性及细胞毒性同时在篮选的内容之列. 但正是由于这一点, 像其他细胞水平的篮选方法一样, 小分子通透性往往影响其活性的检测, 同时样品的用量也较大. 尽管蛋白-蛋白的相互作用在各种疾病中起重要作用, 但目前以此为 模型篮选活性小分子还未得到广泛的应用 ${ }^{[3]}$. 反双杂交系统在观念上完全适应高通量快速篮 选的需要, 随着人类基因组学及蛋白质组学研究的进展, 越来越多的与疾病相关的蛋白间相 互作用的阐明必将促进反双杂交系统在活性小分子篮选中的推广与发展.

\section{2 靶蛋白的鉴定}

到目前为止, 大约每 1000 个人体蛋白中只有一个成为药物篮选的靶蛋白. 现在 $90 \%$ 以 上的新药是由大量化合物通过这些靶蛋白的篩选而获得的. 据估计, 人体至少应有 200000 个 蛋白可以作为药物篮选的靶蛋白 ${ }^{[2]}$. 特别是进入后基因组时代, 大量的靶蛋白易于得到, 但如 何确定其活性或功能仍是利用其进行药物篮选的前提. 因此, 寻找和确定与疾病相关的靶蛋 白，进而利用这些靶蛋白来筛选新的活性化合物有很大的发展潜力.

自 20 世纪 80 年代中期, 人们就开始利用分离到的活性天然产物来鉴定靶蛋白, 主要包 括利用一些特殊的生化性质 ${ }^{[28,29]}$ 及细胞篮选 ${ }^{[30]}$ 等, 而亲和色谱 ${ }^{[31 ~} 400$ 是最常用的方法. 这几种 方法的一个共同点是在蛋白水平上篮选, 由于很多靶蛋白在生物体中含量较少, 常常给分离 鉴定造成很大困难. 最近, 几种新方法的出现可望解决这个问题.

\section{1 三杂交系统}

三杂交系统(three-hybrid system) $)^{[41]}$ 是近几年在酵母双杂交技术 ${ }^{[21,22]}$ 的基础上发展的由活 性小分子鉴定靶蛋白的方法. 其基本原理与酵母双杂交类似(图 5), 只是在“钩”与“鱼”之间加 了“饵”构成三杂交中的 3 个组分：其中的“饵”是一种修饰过的活性小分子, 是由活性小分子 和配体 A 连接而成; “钩” 是由配体 A 的受体蛋白与 DB 融合而成; “鱼” 是由 cDNA 库中的蛋白 融合在 $\mathrm{AD}$ 上构成. 当待篮选靶组织的 $\mathrm{cDNA}$ 库中的某一蛋白与小分子相互作用时报告基因 的转录被启动, 这时细胞可被明显检测. 用地塞米松 (一种甾体激素)修饰后的 FK506 作为饵, 糖皮质激素受体与 DNA 结合蛋白融合作为钩, 采用该方法从人 cDNA 库中篮选到 FK506 的 靶蛋白 FKBP-12 $2^{[41]}$. 最近在哺乳动物组织细胞中采用三杂交系统篮选活性小分子的靶蛋白已 获得成功 ${ }^{[42]}$.

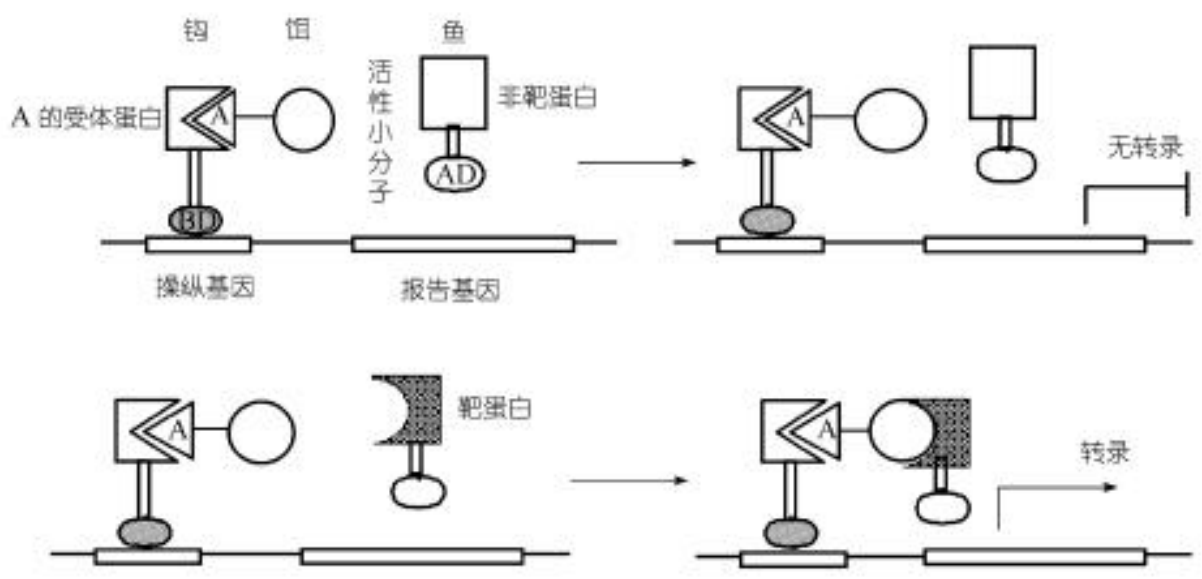

图 5 三杂交系统由活性小分子鉴定靶蛋白原理示意图 


\section{2 功能表达克隆}

与其他方法相比，通过功能表达克隆(functional expression cloning)由活性小分子鉴定靶 蛋白具有其独特性. 首先, 活性小分子不需衍生或修饰; 其次所用的表达系统可以是哺乳动 物或人的组织细胞, 因此表达出的靶蛋白通常是经过正常修饰的, 这一点对于膜蛋白尤其重 要. 该方法一个前提条件是必须明确活性小分子作用的靶组织及小分子作用后引起的靶组织 细胞功能的改变, 以此选择表达体系及确定检测手段. 辣椒素(capsacin)是红辣椒的活性成分, 研究推测其作用于神经元的某受体而产生热疼感. 生理研究发现辣椒素可导致 $\mathrm{Ca}^{2+}$ 大量流入 这些神经元细胞 ${ }^{[43,44]}$, 而 $\mathrm{Ca}^{2+}$ 大量流入细胞可以通过一定的方法 ${ }^{[45]}$ 结合显微观察检测. 在此 基础上, Clapham 等人 ${ }^{[46 ~ 48]}$ 等人采用功能表达克隆方法由辣椒素篮选到其受体蛋白(图 6), 首 先建立神经元细胞的 cDNA 库, 将其分为几个亚库, 分别转染人胚肾细胞衍生株 HEK293(本 身不含神经元细胞的基因); 然后用辣椒素分别与之相互作用, 通过苂光显微检测, 显示信 号的即是含有受体蛋白的亚库. 将该亚库进一步分为几个次级亚库, 采用相同方法进行篮选, 如此循环往复. 每经过一轮䇻选, 库中显示信号的克隆比例得到一次提高, 直至库中所用克 隆均显示信号说明篮选完成. 进一步的研究证明该靶蛋白是一个热伤害敏感的非选择性阳离 子通道, 可以直接被辣椒素活化. 这就揭示了辣椒产生热疼感的本质, 从分子水平阐明了小 分子参与的该生命活动的调节过程.

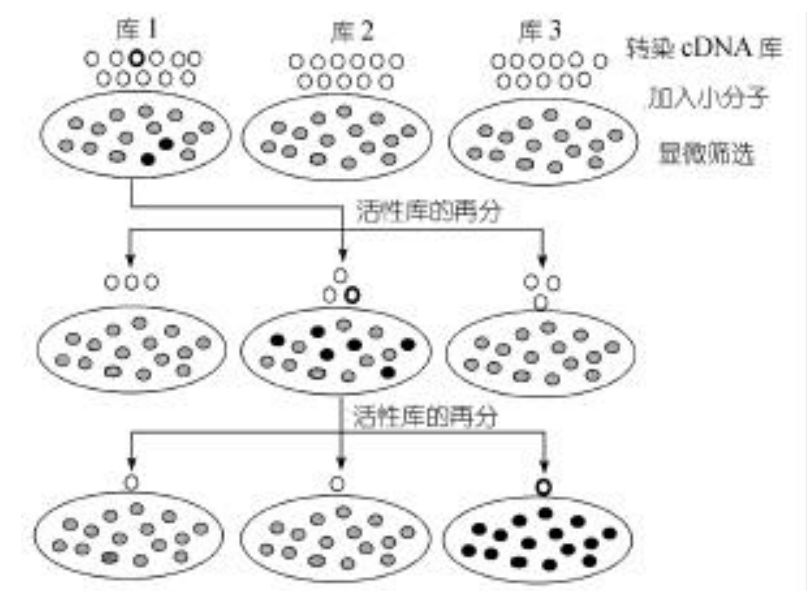

图 6 功能表达克隆由活性小分子鉴定靶蛋白原理示意图

\section{3 药物印迹}

药物印迹(drug-western) 是以免疫印迹 为基础, 采用标记的活性小分子(或药物)为 探针鉴定靶蛋白及其基因的有效方法. 其基 本原理 ${ }^{[48,49]}$ 如图 7 所示. 首先采用 $\lambda$ 噬菌体 构建靶组织的 cDNA 库, 转染大肠杆菌, 这 样培养平板上每一个菌斑中均表达 cDNA 库中的一种蛋白; 然后把这些蛋白转移到硝 酸纤维膜上, 将经牛血清蛋白(BSA)交联的

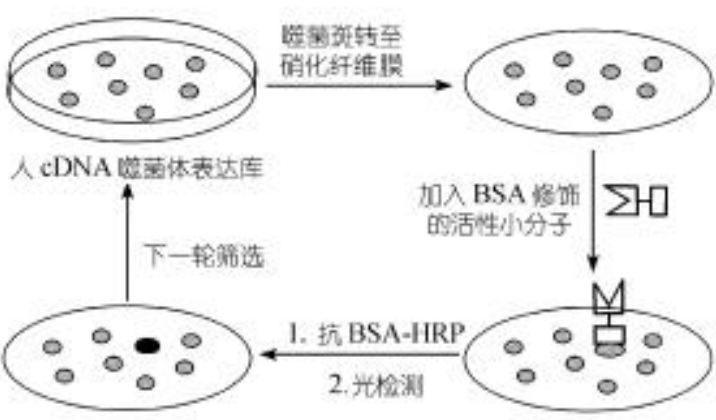

图 7 药物印迹由活性小分子鉴定靶蛋白原理 示意图 
活性小分子与转移在膜上的蛋白充分作用; 经过适当淋洗后, 用 BSA 抗体-辣根过氧化酶 (HRP)与之作用, 经相应的化学发光方法检测. Tanaka 等人 ${ }^{[49]}$ 采用该方法, 用一种抗肿瘤的磺 胺药从人胎盘 cDNA 库的 200 万个菌落中篮选到 10 个与之相互作用, 其中 6 个与已知的蛋 白毫无同源性, 剩余 4 个分别是生长激素, 促性腺激素, 转录因子 NF-Y 的 B 亚基和胸腺素 $\beta$ 10. 进一步研究发现后面两个正是该药物发挥作用的靶蛋白.

上述 3 种由活性小分子篮选靶蛋白的方法与传统方法相比有很大改进. 由于结合了基因 的表达克隆, 大大提高了检出低丰度靶蛋白的能力; 同时由于与基因相关联, 也加快了由蛋 白到基因的速度. 但严格地说, 3 种方法仍是蛋白水平上篮选的改进. 因此, 一步完成活性小 分子到靶蛋白基因水平的鉴定将更适应化学基因学发展的要求.

\section{4 展示克隆}

展示克隆(display cloning)是综合了亲和层析、噬菌体展示和聚合酶链式反应(PCR) 技术 发展的一种由活性小分子直接鉴定细胞中靶蛋白及其编码基因的新方法. 该方法一般需要 5 个基本步骤(图 8) ${ }^{[50]}$ : (1) 制备生物素标记的活性小分子化合物的探针, 利用生物素结合蛋白 与生物素的亲和作用将该探针固定在固相支持物上形成柱体; 同时构建待篮选靶组织细胞的 cDNA 噬菌体蛋白展示库, 并通过柱体. (2) 洗柱. 由于噬菌体表面所展示的靶蛋白与小分子 的相互作用, 展示靶蛋白的噬菌体被牢牢吸附, 而展示非靶蛋白的噬菌体被洗脱. (3) 生物素 淋洗. 将生物素标记的活性小分子连同结合的靶蛋白-噬菌体一起由亲和柱洗下. (4) 洗下的踨 菌体转染大肠杆菌,含靶蛋白的基因的噬菌体得到大量扩增. (5)分析鉴定. 采用 PCR 方法对扩 增的噬菌体中靶蛋白(或靶蛋白结构域)的基因进行扩增、分析, 必要的情况下进行第 2 轮篮选.

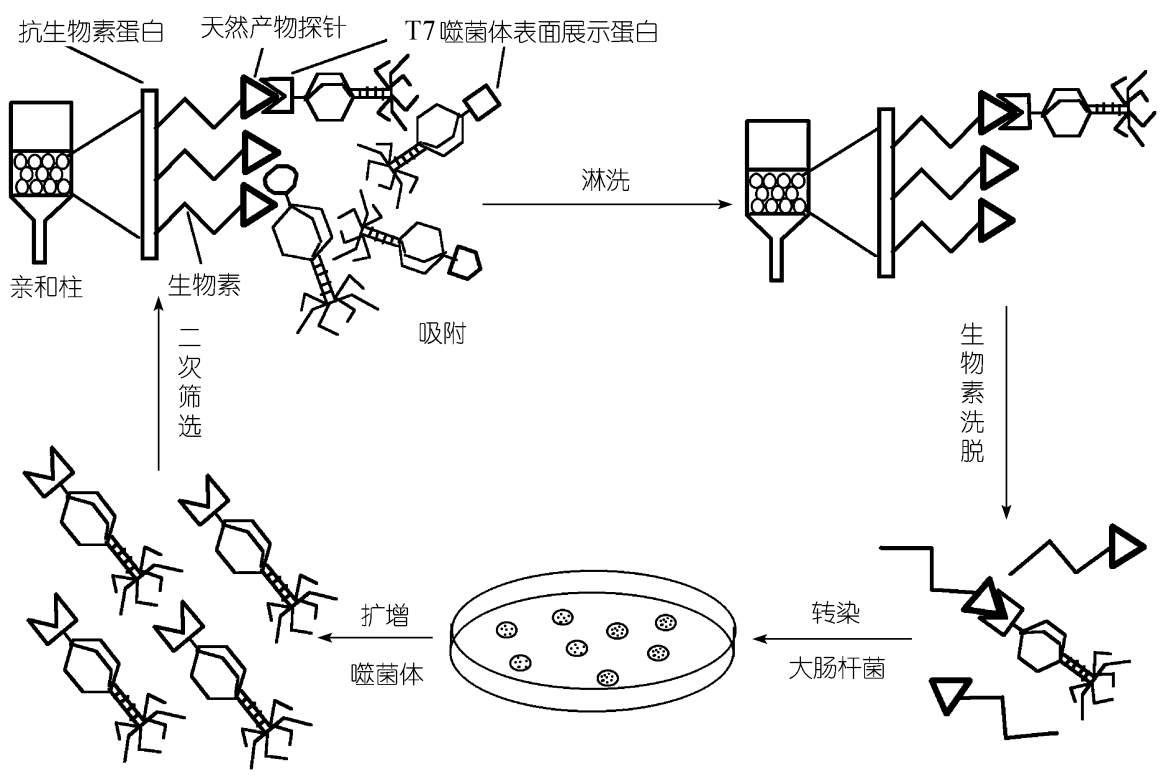

图 8 展示克隆由活性小分子鉴定靶蛋白原理示意图

由于结合了喍菌体展示和 PCR 方法, 该方法减少了多步篮选造成的高背景及不确定性等 问题, 可以一步完成由活性小分子到靶蛋白基因的克隆; 理论上不仅可以获得与天然产物作 
用最强的靶蛋白, 而且同时还能得到一系列与天然产物相互作用的靶蛋白或靶蛋白的结构域. 利用该方法, Sche 等 ${ }^{[50]}$ 成功地实现了由免疫抑制剂 FK506 直接篮选出 FK506 结合蛋白 (FKBP12)基因. 他们利用生物素标记的 FK506 分子为探针, 选用新型的 T7 噬菌体展示系统 ${ }^{[51]}$ 构建人脑的 cDNA 展示库. 该系统中由于外源的待展示基因插入在包被蛋白 $\mathrm{cp} 10$ 的 C 端, 不 影响正常表达及展示, 同时该展示系统具有良好的分泌性. 经过洗柱、淋洗及转染之后, 根据 噬菌体已知的 DNA 序列设计 PCR 引物扩增 $\mathrm{CDNA}$ 的插入片段进行分析. 篮选前扩增产物杂 乱无章; 经过第 1 轮䇥选, 可以得到特异性很强的几组, 说明这些基因所编码的蛋白或结构 域与该天然产物有或强或弱的相互作用; 经过第 2 轮篮选, 可以得到单一的扩增产物, 经 DNA 顺序分析表明与 FKBP12 的编码基因一致,说明该基因编码的蛋白与 FK506 有更强的相互作用.

展示克隆方法一经提出立即引起了人们极大的兴趣 ${ }^{[52]}$, 虽然目前还没有其他成功范例的 报道, 但无论从创新性和实用性方面考虑, 该方法在观念上都不失为一种现代分子生物学与 有机化学的完美结合, 它将为人们由活性小分子到靶蛋白的篮选提供一种崭新的思路与手段. 但是该方法对展示系统的要求较高, 因为生物体中许多靶蛋白要经过翻译后加工, 如磷酸 化、酰基化及糖基化等, 另外多亚基蛋白的组装也是展示系统面临的挑战之一. 因此, 发展新 型的、对展示蛋白能够进行修饰及组装且具有普适性的展示系统是该方法未来的发展方向之

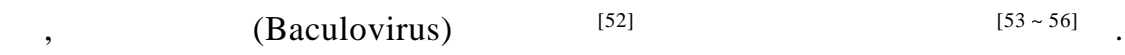

2.5 以基因组为基础的靶蛋白鉴定及小分子参与的生物大分子调控

DNA 芯片及其相关技术的发展及在鉴定靶蛋白、了解小分子参与方面的应用适应了人 类基因组学、蛋白质组学及化学基因组学发展的需要, 因为对小分子作用后的细胞进行基因 组水平的分析, 探明小分子对基因转录、表达的影响是具有普适性的方法之一, 同时可以提 供有关代谢过程及基因组方面的信息 ${ }^{[57]}$. 其最基本的实验是检测活性小分子作用后靶组织细 胞中 mRNA 丰度的增加或减少, 最基本的工具是 DNA 芯片. 首先, 分别从靶组织细胞及小分 子处理后的靶组织细胞中提取 mRNA, 标记, 然后将之与 cDNA 阵列(或芯片) ${ }^{[58]}$ 或寡核苷酸 芯片 ${ }^{[59}$ 杂交. 通过检测已知基因表达的变化, 可以阐明小分子参与所引发的一系列代谢及调 控过程; 而未知基因表达的变化可以以不同的小分子为探针, 通过指纹对比研究基因、蛋白 的功能.

最近, 采用 DNA 芯片技术, 将酵母基因组与小分子参与的转录表达谱(transcriptional or expression profiling)相结合, 可以用来鉴定靶蛋白. 原理 ${ }^{[48]}$ 如图 9 所示. 首先构建缺失某一假 定靶蛋白基因的酵母突变株, 将野生型及突变株分别用活性小分子处理, 提取处理前后的 mRNA, 反转录为苂光标记的 cDNA; 然后与 DNA 芯片杂交以确定每一样品的 mRNA 丰度. 在第 1 组实验中, 对野生型酵母经小分子处理前后进行比较 (A 比 B); 第 2 组实验中, 未经小 分子处理的野生型酵母与突变株酵母进行比较 ( $\mathrm{A}$ 比 $\mathrm{C}$ ). 若缺失的是活性小分子的靶蛋白基因, 则两组实验的转录表达谱相似; 反之若缺失的不是靶蛋白基因, 二者转录表达谱明显不同. 如 用 FK506 处理后酵母细胞的转录分布图与缺失钙调磷酸酶(Calcineurin)后的酵母突变株转录 表达谱十分类似, 进一步证明钙调磷酸酶确是 FK506 的靶蛋白之一 ${ }^{[60]}$. 该方法的一种改进法 是将缺失某一假定靶蛋白基因的酵母突变株经活性小分子作用前后的转录表达进行比较 $(\mathrm{C}$ 比 D), 若缺失的是靶蛋白基因, 则转录表达没有变化, 图上无信号; 反之若缺失的不是靶蛋白基 
因, 二者变化明显. 当用 FK506 处理缺失钙调 磷酸酶后的酵母突变株, 发现其转录表达与处 理前相比有几处变化, 说明在酵母细胞中还有 其他靶蛋白 ${ }^{[60]}$. 该方法最大的优越性是活性小 分子不需衍生化, 且可以揭示小分子参与的代 谢及调控过程, 研究基因、蛋白的功能. 但对 靶组织有严格的要求, 即靶组织中的蛋白必须 是可以通过基因缺失而完全失活. 目前满足这 一要求的有细菌、酵母、果蝇、线虫及小鼠等.

最近 Stockwell 等人 ${ }^{[6] 1}$ 综合多种篮选方法 䇻选活性小分子, 再进一步由活性小分子鉴定 靶蛋白, 研究小分子参与的调控, 是化学基因 学取得成功的明显例子. 首先在细胞水平对 16000 种化合物进行了初篮 ${ }^{[61,62]}$, 得到 4 种活 性小分子; 进一步以细胞印迹篮选它们, 从中 获得两个活性化合物. 分别提取这两种活性分 子处理前后酵母细胞的 mRNA, 反转录为苂光 标记的 cDNA, 与酵母基因的 DNA 芯片杂交, 研究其差异表达谱的变化. 结果发现其中一个
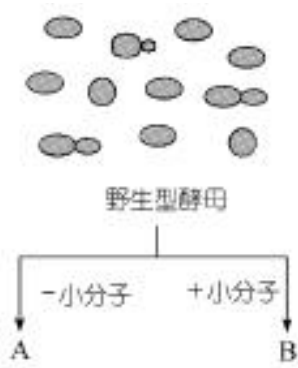

基因 $\mathrm{X}$ 绊码跑蛋白
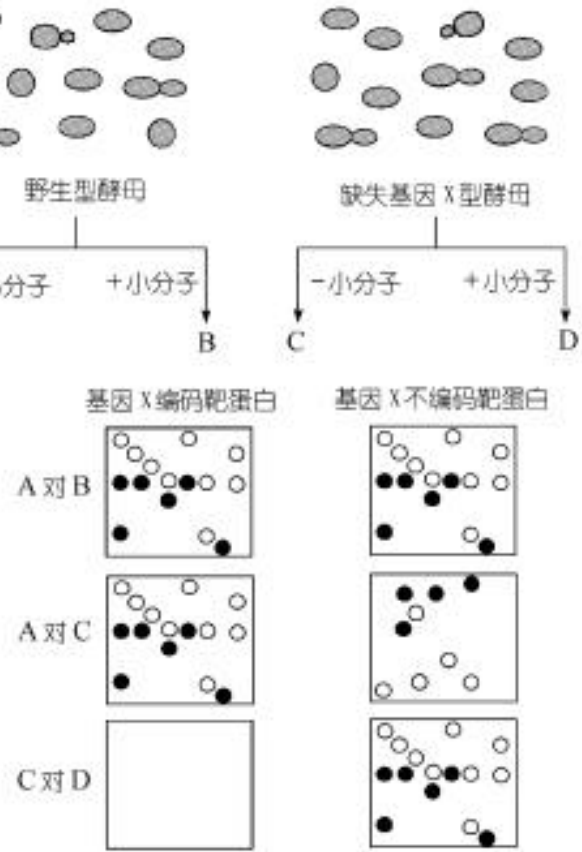

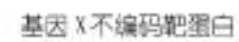
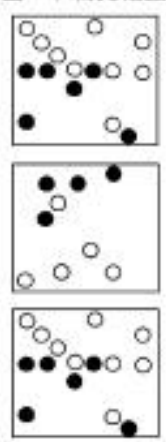

图 9 转录表达谱与基因组相结合鉴定靶蛋白 若前者信号强显示红色，在图中以圈表示; 后者信号强显 示绿色, 以点表示; 二者信号相同无显示 分子对酵母基因表达没有明显影响, 另一个活性小分子增加 5 个基因的表达, 其中 3 个是已 知的, 分别编码热休克蛋白 HSP26、锌转运蛋白 ZRT1 及依赖于铜的铁氧化酶 FET3, 在酵母 中它们与维持金属离子的内环境稳定有关; 另外两个新基因 $Y D R 534 C$ 和 YOL155C 可以被细 胞内 $\mathrm{Cu}^{2+}, \mathrm{Fe}^{3+}$ 及 $\mathrm{Zn}^{2+}$ 的含量所调控; 其编码的蛋白还有待于进一步研究.

\section{3 适应于化学基因学的靶蛋白与活性小分子篮选一小分子印迹}

随着新技术的引 ${ }^{[63,64]}$ ，化学家结合固相合成方法 ${ }^{[65]}$ 合成了大量天然产物的类似物作为 篮选蛋白配体的小分子库, 但是如何充分利用已有的各种靶蛋白来快速篮选其中可能含有的 所有活性配体及利用已有的活性小分子来快速确定某一细胞、组织或生物体中所有的小分子 参与的蛋白调控仍是问题的关键. 最近, 借鉴 DNA 芯片技术, 人们发展了一种称为小分子印 迹(small molecule printing, SMP)的技术来篮选靶蛋白的小分子配体及研究小分子参与的蛋白 调控 ${ }^{[66]}$. 首先, 将应用组合化学在固相载体上合成的有机小分子分别溶解在适当溶剂中, 采 用高精度的机器人 ${ }^{[58]}$ 将 $1 \mathrm{~nL}$ 含有每一种有机小分子的样品溶液精确定位点样于一块经过处 理的载片上. 所有化合物均含有共同的连接反应官能团, 可以通过共价键的形式将小分子固 定在载片上, 这样每一种有机小分子就被固定在特定的位置上形成高密度的小分子阵列(目前 可以达到 1000 个点 $/ \mathrm{cm}^{2}$ ). 在此基础上, 这些小分子可以依次被多种标记的靶蛋白分别进行篮 选，当某一小分子与某种靶蛋白发生结合时可通过荧光偶联检测. 该方法选择性好, 灵敏度高, 不仅可用于靶蛋白配体的有效筛选, 结合亲和层析还可用于靶蛋白的纯化及测定小分子配体 与靶蛋白的结合动力学. 目前 SMP 在 3 类已知的靶蛋白配体篎选中得到初步验证 ${ }^{[66]}$. 该方法 
另一个明显的优势是有机小分子用量少(每一个直径为 $425 \mu \mathrm{m}$ 的聚苯乙烯固相单体上合成的 量可至少产生 7200 个点), 因此用尽可能多的、功能明确的活性有机小分子依次排列制成的 高密度小分子阵列来篮选任何感兴趣的细胞、组织或生物体中的所有蛋白, 理论上凡是有小分 子参与的对所有靶蛋白的调控均可以检测到, 这对于功能基因组及化学基因学的研究有重要的 意义.

SMP 的提出是现代有机化学、生物学、药物化学及 DNA 芯片技术等现代科技发展的必 然结果, 是一种观念上各学科精髓的高度综合, 适应了人类基因组计划发展的需要. 与其他方 法相结合, 其进一步发展必然为后基因组时代的研究提供有效的工具.

\section{4 展望: 化学基因学与中草药现代化}

当归龙荟丸是中国传统中药, 具有泻火通便的功效 ${ }^{[67]}$, 近来发现可用于治疗慢性白血病, 其中靛玉红(indirubin)是主要的活性成分 ${ }^{[68]}$, 可以抑制 DNA 的合成 ${ }^{[69]}$, 其许多类似物具有良

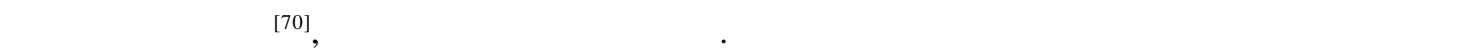
(cyclin-dependent kinases, CDKs) 对细胞周期的起始具有决定性作用, 同时参与细胞周期的分 裂循环 ${ }^{[711}$. 而 CDKs 的化学抑制剂往往具有很好的抗肿瘤活性, 还可用于心血管疾病等的治 ${ }^{{ }^{3}}{ }^{[72]}$. 最近, 德、法、英 3 国科学家在从中国传统中药药用植物中寻找抗肿瘤化合物时, 发 现靛玉红及其类似物可选择性地抑制 CDK2 而阻断细胞的分裂 ${ }^{[73]}$. 他们还通过两种鉦玉红类 似物与 CDK2 复合物的晶体 X 射线衍射分析阐明了抑制剂与 CDK2 的 ATP 结合口袋的相互 作用是该类化合物具有生物活性的根本原因. 这一机理的阐明把人们对中草药的认识提高到 一个新的层次, 同时该靶蛋白模型的确证也为进一步篮选高效低毒的抗肿瘤药物奠定了基础.

中草药是中国人民在长期与疾病作斗争中积累的经验总结, 在中华民族的发展及人民健 康事业中起了重要作用. 但由于中药缺乏系统的现代科学基础研究, 未能进入国际天然药物 市场的主流. 中药在国际市场上未得到广泛承认的主要原因之一是大多数中草药的作用机制 不清楚, 有效成分不明确, 或缺少严格的质量指标. 要使中草药走向世界, 与世界现代医学接 轨, 并打开国际市场, 就必须加强基础研究, 在中草药研究中发展并广泛应用高新技术, 结合 当前人类基因组研究、蛋白质组研究的最新成果, 在细胞、分子及化学键水平上阐明其治疗 的科学原理, 实现中草药现代化. 综合应用有机化学、分子生物学结合现代波谱学等手段建 立并发展研究中草药中活性有机小分子与生物大分子间相互作用、相互识别的高技术, 不仅
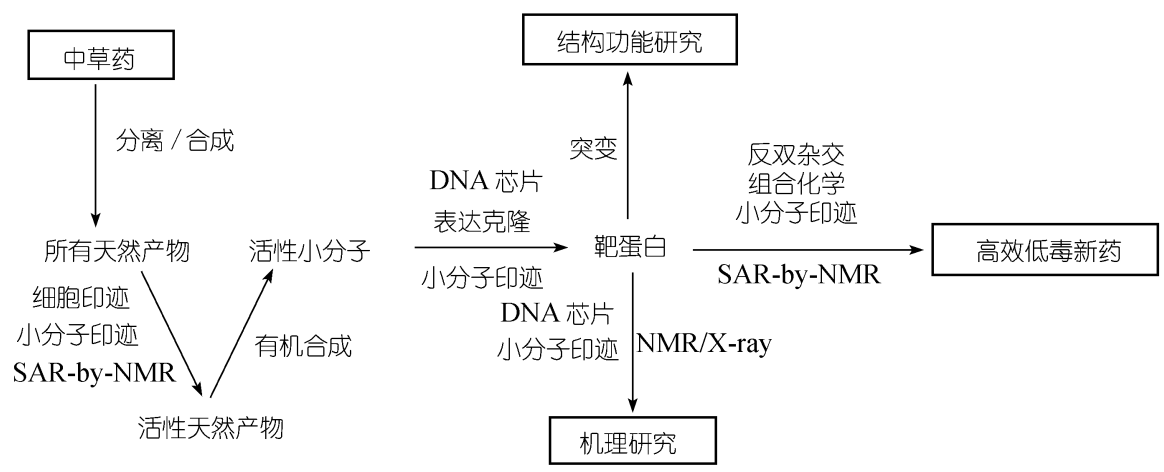

图 10 中草药现代化设想示意图 
可以阐明许多中草药的作用机制, 促进中草药现代化, 而且对于开发具有我国自主知识产权 的新药具有重要意义(图 10).

随着人类基因组计划的进展, 蛋白质组学时代的到来, 一个新的融合有机化学、分子生 物学, 以研究活性有机小分子和靶蛋白相互作用、寻找和篮选新药为目的的化学基因学正在 形成. 如果人类基因组计划的目标是“分析人体所有基因”, 蛋白组学研究的目标是“确定每一 个基因表达产物及其功能”, 那么化学基因学研究的最终目标就是“鉴定每一个基因表达产物 的小分子参与物” ${ }^{[74]}$. 由于有机化学、分子生物学、基因组学及蛋白质组学等研究提供了充足 的小分子及靶蛋白, 人们可以根据需要随意地以活性小分子为探针鉴定靶蛋白, 通过它们之间 的相互作用来研究其结构与功能, 从本质上理解小分子对生命活动调节、作用的机理; 同时 结合组合化学, 利用靶蛋白来进一步篮选更为有效的药物先导化合物. 这样人们对于生命的理 解必然进入一个新的层次, 因而有可能从更深刻理解分子相互作用原理上控制严重威胁人类健 康的疾病.

\section{参考文献}

1 Mitchison T J. Towards a pharmacological genetics. Chemistry \& Biology, 1994, 1: $3 \sim 6$

2 Vastag B. "Chemical Genetics" speeds up drug discovery. J National Cancer Institute, 1998, 23: 1771

3 Vidal M, Endoh H. Prospects for drug screening using the reverse two-hybrid system. Tibtech, 1999, 17: $374 \sim 381$

4 Crews C M, Splittgerber U. Chemical genetics: exploring and controlling cellular processes with chemical probes. Trends Biochem Sci, 1999, 24: $317 \sim 320$

5 Stockwell B R, Haggarty S J, Schreiber S L. High-throughput screening of small molecules in miniaturized mammalian cell-based assays involving post-translational modifications. Chemistry \& Biology, 1999, 6: $71 \sim 83$

6 Mayer T U, Kapoor T M, Haggarty S J, et al. Small molecular inhibitor of mitotic spindle bipolarity identified in a phenotype-based screen. Science, 1999, 286: $971 \sim 974$

7 Haggarty S J, Mayer T U, Miyamoto D T, et al. Dissecting cellular processes using small molecules: identification of colchicine-like, taxol-like and other small molecules that perturb mitosis. Chemistry \& Biology, 2000, 7: $275 \sim 286$

8 Borman S. Anticancer research pans out: unique agent hones in a different target than previous antimitotic drugs like taxol. C\&EN, 1999, November 1: 6

9 Strobel C. ICCB discovers first in new class of mitosis inhibitors. http://www.med.harvard.edu/publications/Focus/oct 29_1999/cell_biology.html

10 Drews J. Drug discovery: a historical perspective. Science, 2000, 287: 1960 1964

11 麻锦彪, 吴厚铭. 发现生物大分子高亲和性配体的新方法—SAR-by-NMR. 化学进展, 1999, 11: 265 274

12 Shuker S B, Hajduk P J, Meadows R, et al. Discovering high-affinity ligands for proteins: SAR by NMR. Science, 1996, 274: $1531 \sim 1534$

13 Medek A, Hajduk P J, Mack J, et al. The use of differential chemical shifts for determining the binding site location and orientation of protein-bound ligands. J Am Chem Soc, 2000, 122: $1241 \sim 1242$

14 Rouhi M. Characterizing how ligands bind to proteins by NMR. C\&EN, 2000, Feb 21: $30 \sim 31$

15 Hajduk P J, Sheppard G, Netteshein D G, et al. Discovery of potent nonpeptide inhibitors of stromelysin using SAR by NMR. J Am Chem Soc, 1997, 119: $5818 \sim 5827$

16 Hajduk P J, Dinges J, Miknis G F, et al. NMR-based discovery of lead inhibitors that block DNA binding of the human papillomavirus E2 protein. J Med Chem, 1997, 40: $3144 \sim 3150$

17 Hajduk P J, Zhou M -M, Fesik S W. NMR-based discovery of phosphotyrosine mimetics that bind to the LCK SH2 domain. Bioorg Med Chem Lett., 1999, 9: $2403 \sim 2406$

18 Hajduk P J, Dihges J, Achkeryantz J M, et al. Novel inhibitors off Erm Methyl-transferase from NMR and parallel synthesis. J Med Chem, 1999, 42: $3852 \sim 3859$

19 Hajduk P J, Gerfin T, Boehlen J -M, et al. High-throughput nuclear magnetic resonance-based screening. J Med Chem, 1999, 
42: $2315 \sim 2317$

20 Cochran A G. Antagonists of protein-protein interactions. Chemistry \& Biology, 2000, 7: R85 R94

21 Fields S, Song O. A novel genetic system to detect protein-protein interactions. Nature, 1989, 340: $245 \sim 246$

22 Vidal M, Legrain P. Survey and summary: yeast forward and reverse " $n$ "-hybrid systems. Nucleic Acids Res, 1999, 27: $919 \sim 929$

23 秦宝明, 罗述金, 米志勇, 等. 酵母双杂合系统的改进和发展. 生物工程进展, 1998, 18:2 9

24 Vidal M, Brachmann R, Fattaey A, et al. Reverse two-hybrid and one-hybrid systems to detect dissociation of proteinprotein and DNA-protein interactions. Proc Natl Acad Sci USA, 1996, 93: 10315 10320

Huang J, Schreiber S L. A yeast genetic system for selecting small molecule inhibitors of protein-protein interactions in nanodroplets. Proc Natl Acad Sci USA, 1997, 94: 13396 13401

26 Wang T, Donahoe P K, Zervos A S. Specific interaction of type I receptors of the TGF- $\beta$ family with the immunophilin FKBP-12. Science, 1994, 265: 674 676

27 Young K, Lin S, Sun L, et al. Identification of a calcium channel modulator using a high throughput yeast two-hybrid screen. Nature Biotechnol, 1998, 16: 946 950

28 Shoyab M, Boaze R Jr. Isolation and characterization of a specific receptor for biologically active phorbol and ingenol esters. Arch Biochem Biophys, 1984, 234: 197 205

29 Miesfeld R, Okret S, Wikstrom A C, et al. Characterization of a steroid hormone receptor gene and mRNA in wide-type and mature cells. Nature, 1984, 312: $779 \sim 781$

30 Monks A, Scudiero D A, Johnson G S, et al. The NCl anti-cancer drag screen: a smart screen to identify effectors of novel targets. Anticancer Drug Des, 1997, 12: $533 \sim 541$

31 Harding M W, Galat A, Uehling D E, et al. A receptor for the immunosuppressant FK506 is a cis-trans peptidylprolyl isomerase. Nature, 1989, 341: $758 \sim 760$

32 Handschumacher R E, Harding M W, Rice J, et al. Cyclophilin: a specific cytosolic binding protein for cyclosporin A. Science, 1984, 226: $544 \sim 547$

33 Fenteany G, Standaert R F, Lane W S, et al. Inhibition of proteasome activities and subunit-specific amino-terminal threonine modification by lactacystin. Science, 1995, 268: $726 \sim 731$

34 Crews C M, Lane W S, Schreiber S L. Didemnin binds to the protein palmitoyl thioesterase responsible for infantile neuronal ceroid lipofuscinosis. Proc Natl Acad Sci USA, 1996, 93: 4316 4319

35 Hung D T, Chen J, Schreiber S L. (+)-Discodermolide binds to microtubules in stoichiometric ratio to tublin dimers, blocks taxal binding and results in mitotic arrest. Chem Biol, 1996, 3: $287 \sim 293$

36 Taunton J, Hassig C A, Schreiber S L. A mammalian histone deacetylase related to the yeast translational regular Rpd3p. Science, 1996, 272: $408 \sim 411$

37 Sin N, Meng L, Wang M Q, et al. The anti-angiogenic agent fumagillin covalently binds and inhibits the methionine aminopeptidase, MetAP-2. Proc Natl Acad Sci USA, 1997, 94: 6099 6103

Griffith E C, Su Z, Turk B E, et al. Methionine aminopeptidase (type 2) is the common target for angiogenesis inhibitors AGM-1470 and ovalicin. Chem Biol, 1997, 4: $461 \sim 471$

39 Sin N, Meng L, Auth H, et al. Eponemycin analogues: syntheses and use as probes of angiogenesis. Bioorg Med Chem, 1998, 6: $1209 \sim 1217$

40 Chen J K, Lane W S, Schreiber S L. The identification of myricin-binding proteins. Chem Biol, 1999, 6: $221 \sim 235$

41 Licitra E J, Liu J O. A three-hybrid system for detecting small ligand-protein receptor interactions. Proc Natl Acad Sci, 1996, 93: $12817 \sim 12821$

42 Liberles S D, Diver S T, Austin D L, et al. Inducible gene expression and protein translocation using nontoxic ligands identified by a mammalian three-hybrid screen. Proc Natl Acad Sci, 1997, 94: $7825 \sim 7830$

Wood J N. Capsaicin-induced ion fluxes in dorsal root ganglion cells in culture. J Neurosci, 1988, 8: $3208 \sim 3220$

Dray A, Forbes C A, Burgess G M. Ruthenium red blocks the capsaicin-induced increase in intracellular calcium and activation of membrane currents in sensory neurones as well as the activation of peripheral nociceptors in vitro. Neurosci Lett, 1990, 110: $52 \sim 59$

45 Tsien R Y. Fluorescent probes of cell signaling. Annu Rev Neurosci, 1989, 12: $227 \sim 253$

46 Clapham D E. Some like it hot: spicing up ion channels. Nature, 1997, 389: $783 \sim 784$ 
47 Caterina M J, Schumacher M A, Tominaga M, et al. The capsaicin receptor — a heat-activated ion channel in the pain pathway. Nature, 1997, 389: 816 824

48 King R W. Chemistry or biology: which comes first after the genome is sequenced? Chem Biol, 1999, 6: R327 R333

49 Tanaka H, Ohshima N, Hidaka H. Isolation of cDNAs encoding cellular drug-binding proteins using a novel expression cloning procedure: drug-western. Mol Pharmacol, 1999, 55: 356 363

50 Sche P P, Mckenzie K M, White J D, et al. Display cloning: functional identification of natural product receptors using cDNA-phage display. Chem Biol, 1999, 5: $707 \sim 716$

51 Rosenberg A, Mierendorf R. T7 select phage display system: a powerful new protein display system based on bacteriophage T7. Novations, 1996, 6: $1 \sim 6$

52 Borman S. Protein targets of bioactive natural products probed. C\&EN, 1999, Oct 4: $33 \sim 34$

53 Cho B K, Kieke M C, Boder E T, et al. A yeast surface display system for the discovery of ligands that trigger cell activation. J Immunol Methods, 1998, 220: 179 188

54 Shusta E V, Kieke M C, Parke E, et al. Yeast polypeptide fusion surface display levels predict thermal stability and soluble secretion efficiency. J Mol Biol, 1999, 292(5): $949 \sim 956$

55 Lin Cereghino G P, Cregg J M. Applications of yeast in biotechnology: protein production and genetic analysis. Current Opinion in Biotechnology, 1999, 10(5): $422 \sim 427$

56 Kieke M C, Shusta E V, Boder E T, et al. Selection of functional T cell receptor mutants from a yeast surface-display library. Proc Natl Acad Sci USA, 1999, 96: $5651 \sim 5656$

Brown P O, Botstein D. Exploring the new world of the genome with DNA microarrays. Nat Genet, 1999, 21: $33 \sim 37$

58 Schena M, Shalon D, Davis R W, et al. Quantitative monitoring of gene expression patterns with a complementary DNA microarray. Science, 1995, 270: $467 \sim 470$

59 Lockhart D J, Brown E L. Expression monitoring by hybridization to high-density oligonucleotide arrays. Nat Biotechnol, 1996, 14: $1675 \sim 1680$

60 Marton M J, Friend S H. Drug target validation and identification of secondary drug target effects using DNA microarrays. Nat Med, 1998, 4: $1293 \sim 1301$

61 Stockwell B R, Hardwick J S, Tong J K, et al. Chemical genetic and genomic approaches reveal a role for copper in specific gene activation. J Am Chem Soc, 1999, 121: $10662 \sim 10663$

62 Stockwell B R, Schreiber S L. Probing the role of homomeric and heteromeric receptor interactions in TGF- $\beta$ signaling using small molecule dimerizers. Current Biology, 1998, 8: $761 \sim 770$

63 Lam K S, Salmon S E, Hersh E M, et al. A new type of synthetic peptide library identifying ligand-binding activity. Nature, 1991, 354: $82 \sim 84$

64 Nestler H P, Bartlett P A, Still W C. A general method for molecular tagging of encoded combinatorial chemistry libraries. J Org Chem, 1994, 59: 4723 4724

65 Tan D S, Foley M A, Shair M D, et al. Stereoselective synthesis of over two million compounds having structural features both reminiscent of natural products and compatible with miniaturized cell-based arrays. J Am Chem Soc, 1998, 120: $8565 \sim 8566$

MacBeath G, Koehler A N, Schreiber S L. Printing small molecules as microarrays and detecting protein-ligand interaction en masse. J Am Chem Soc, 1999, 121: $7967 \sim 7968$

67 Chinese Pharmacopoeia, Vol 1. Beijing: People's Health Publisher, 1995

68 Chen DH, Xie J X Chemical constituents of traditional Chinese medicine Qing Dai.Chinese Trad Herb Drugs, 1984,15: 6 8

69 Chang H M, But P P H. Pharmacology and Application of Chinese Materia Medica, Vol 2. Teaneck N J, ed. Singapore: World Scientific Pub Co, 1987

70 Gu Y C, Li G L, Yang Y P, et al. Synthesis of some halogenated indirubin derivatives. Acta Pharm Sin, 1989, 24: 629 632 Morgan D. Cyclin-dependent kinases: engines, clocks, and microprocessors. Annu Rev Cell Dev Biol, 1997, 13: $261 \sim 291$ Meijer L, Kim S H. Chemical inhibitors of cyclin-dependent kinases. Methods Enzymol, 1997, 283: $113 \sim 128$

73 Hoessel R, Leclerc S, Endicott J A, et al. Indirubin, the active constituent of a Chinese antileukaemia medicine, inhibits cyclin-dependent kinases. Nature Cell Biol, 1999, 1: $60 \sim 67$

74 Schreiber S L. Chemical genetics resulting from a passion for synthetic organic chemistry. Bioorganic \& Medicinal Chemistry, 1998, 6: $1127 \sim 1152$

(2000-01-26 收稿, 2000-04-24 收修改稿) 